\title{
Effect of Pregabalin on Perioperative Headache in Patients with Aneurysmal Subarachnoid Hemorrhage: A Randomized Double-Blind, Placebo-Controlled Trial
} \author{
Unnikrishnan Prathapadas ${ }^{1}$ Ajay Prasad Hrishi ${ }^{1}$ \\ ${ }^{1}$ Division of Neuroanaesthesia, Sree Chitra Tirunal Institute for \\ Medical Sciences and Technology, Thiruvananthapuram, Kerala, \\ India \\ ${ }^{2}$ Department of Neurosurgery, Sree Chitra Tirunal Institute for \\ Medical Sciences and Technology, Thiruvananthapuram, Kerala, \\ India
}

Karen Ruby Lionel ${ }^{1}$ Manikandan Sethuraman ${ }^{1} \quad$ Mathew Abraham $^{2}$ Smita Vimala

\begin{abstract}
Address for correspondence Karen Ruby Lionel, DM, Division of Neuroanaesthesia, Sree Chitra Tirunal Institute for Medical Sciences and Technology, Thiruvananthapuram, Kerala, India

(e-mail: kanjacob@gmail.com).
\end{abstract}

J Neurosci Rural Pract 2019;10:438-443

\begin{abstract}
Background Patients with acute aneurysmal subarachnoid hemorrhage (aSAH) experience excruciating headache that is difficult to manage in resource-constrained settings. Pregabalin's ( $\beta$-isobutyl-GABA) analgesic, antiepileptic, and antiemetic properties make it an attractive adjuvant in pain management for these patients.

Methods We conducted a double-blind, placebo-controlled, randomized clinical trial on 40 aSAH patients undergoing aneurysmal clipping to assess the effect of perioperative pregabalin in decreasing perioperative headache, anesthetic, and opioid requirement. Patients received either pregabalin $(75 \mathrm{mg})$ or placebo twice daily soon after admission till 24-hour postoperative, in addition to paracetamol $650 \mathrm{mg}$ thrice daily. Headache assessed using a visual analog scale (VAS) at five time points was compared using a mixed effects regression model.

Results Pain assessed by VAS declined significantly more from the baseline in pregabalin recipients compared with placebo at preinduction ( -3.6 vs. $-1.8 ; p=0.004$ ), 12 -hour ( 4.3 vs. $2.8 ; p=0.014$ ), and 24 -hour postsurgery ( 4.7 vs. $2.9 ; p=0.007$ ), but not at the 6 -hour postoperation ( 4.9 vs. 3.8; $p=0.065$ ). Pregabalin recipients required a lower minimum alveolar concentration of sevoflurane to maintain a prespecified bispectral index of 40 and 60 ( 0.8 vs. $0.9 ; p=0.014)$ and required fewer rescue analge-

\section{Keywords}

- aneurysmal subarachnoid hemorrhage

- aneurysm clipping

- cephalalgia

- pregabalin

- visual analog scale sic doses in the 24 hours following surgery ( 1.8 vs. $3.3 ; p=0.005)$. The intraoperative fentanyl requirement was not significantly different between the groups $(10 \mu \mathrm{g} / \mathrm{kg}$ vs. $11.4 \mu \mathrm{g} / \mathrm{kg} ; p=0.065$ ). There was no significant difference in the sedation scores. Conclusions Pregabalin $75 \mathrm{mg}$ administered twice daily, during the perioperative period, was an effective adjunct in the management of the severe headache experienced by patients with aSAH and decreased the opioid and anesthetic requirement without significantly increasing sedation.
\end{abstract}

\section{Introduction}

Acute aneurysmal subarachnoid hemorrhage (aSAH) is a catastrophic event presenting with excruciating headache often described as a "thunderclap headache." The origin of this pain is thought to be neuropathic as the meninges which are innervated by the branches of the trigeminal nerve are inflamed as a consequence of exposure to blood, resulting in meningism, 
causing severe headache. ${ }^{1-5}$ In this subset of patients, control of pain is of paramount importance as severe pain results in the activation of the sympathetic system, resulting an increase in the blood pressure and an increase in the level of stress hormones. ${ }^{6,7}$ This, in turn, increases the chance of complications, such as rerupture/rebleed, vasospasm, and stress cardiomyopathy which are associated with greater morbidity and mortality. ${ }^{7-9}$

The choice of analgesic and anti-inflammatory agents in resource-constrained settings is limited. Paracetamol is routinely prescribed but provides suboptimal relief of headache, resulting in demand for rescue analgesia. Because of the risk of platelet dysfunction and rebleed, nonsteroidal anti-inflammatory drugs (NSAIDs) are rarely used in these patients, whereas the sedative effects of opioids make it a challenge to detect the deterioration of sensorium, especially in settings with less than optimal resource for monitoring patients.

Pregabalin ( $\beta$-isobutyl-GABA), a lipophilic structural analog of $\gamma$-aminobutyric acid and a potent gabapentinoid, is effective in the treatment of acute pain, as well as chronic neuropathic pain. ${ }^{10}$ Apart from being a principal tool in the armamentarium against chronic neuropathic pain, pregabalin given perioperatively has been effective in decreasing acute postoperative pain and the need for rescue analgesics in varied settings, including elective neurosurgery for brain tumors and spine surgeries. ${ }^{11}$ Being more potent at reducing neuropathic pain than NSAIDs and paracetamol and less likely to obtund sensorium as compared with opioids, pregabalin might be indicated in those with severe pain. ${ }^{12,13}$ In addition, it has antiepileptic, antiemetic, and anxiolytic properties that make it an attractive option in managing pain in patients with aSAH..$^{14,15}$

We tested the hypothesis that pregabalin would reduce the perioperative pain and headache in patients with aSAH in a setting where oral paracetamol is a standard of care analgesic, by assessing the decline in perioperative headache, use of intraoperative analgesia/anesthesia, and the need for rescue analgesics in the perioperative period.

\section{Methods}

Adult patients with the World Federation of Neurosurgical Societies, grades 1 and 2 aSAH presenting with headache and in whom neurosurgical intervention was proposed were recruited after written informed consent. Those received pregabalin in the previous 2 weeks, having known renal or hepatic dysfunction, or having a Glasgow Coma Scale of 14 or lower were excluded from the trial. The clinical trial was approved by the institutional ethics committee and prospectively registered in the Clinical Trials Registry of India (CTRI/2016/10/007363).

In this double-blind, placebo-controlled randomized trial, patients were randomized in a 1:1 ratio to receive either tablet pregabalin $75 \mathrm{mg} \mathrm{BD}$ (bis in die) or its placebo perioperatively from randomization at admission to 24 hours after surgery. Computer-generated block randomization with varying block sizes was used to assign participants to their respective arms. Allocation concealment was achieved by packaging allocation codes in serially numbered opaque envelopes assembled by a neuroanesthetist, not directly involved with the clinical trial. Nursing staff who were blinded to the study assignment of participants dispensed drugs based on a blinded allocation code. Nurses who were trained to assess pain using a visual analog scale (VAS) assessed pain at recruitment (baseline) and at 6, 12, and 24 hours after surgery. Before every administration of the study drug or rescue analgesic, pain was rated on the VAS, and the degree of sedation was noted by the Ramsay Sedation Scale (RSS) by the nursing staff. All patients received eighth hourly tablet paracetamol $650 \mathrm{mg}$ as per the standard protocol of pain management. At each assessment, if the VAS was 4, injection diclofenac $75 \mathrm{mg}$ was administered intravenously as an infusion. VAS score was reassessed 1 hour later and if still found to be 4 , injection tramadol 50 to $100 \mathrm{mg}$ (as determined by the weight of the patient) was administered. The next line analgesic was injection fentanyl at a dose of $2 \mu \mathrm{g} / \mathrm{kg}$ or injection morphine at $0.05 \mathrm{mg} / \mathrm{kg}$ given intravenously. This step ladder for the treatment of pain was followed till the VAS was $<4$. The same protocol for pain management was followed in the postoperative period as well, with injection paracetamol 1-g intravenous (IV) eighth hourly replacing oral paracetamol as the standard of care analgesic.

Before induction of anesthesia, headache was assessed, and the preinduction VAS was documented by the anesthesiologist who was blinded to the study allocation for the participant. Intravenous induction was performed with a titrated sleeping dose of injection propofol of 1.5 to $2 \mathrm{mg} / \mathrm{kg}$ along with injection fentanyl $2 \mu \mathrm{g} / \mathrm{kg}$. Vecuronium $0.1 \mathrm{mg} / \mathrm{kg}$ was the muscle relaxant of choice. An additional dose of injection fentanyl of $2 \mu \mathrm{g} / \mathrm{kg}$ was administered to attenuate the response to the placement of the Mayfield clamp head holder. Scalp block for intraoperative analgesia was not performed on any of our patients. During surgery, the dose of inhalational anesthetic (sevoflurane) was titrated to maintain the bispectral index (BIS) between 40 and 60. A standardized algorithm was used to assess the need for intraoperative opioids, and the number of additional bolus doses of fentanyl per $\mathrm{kg}$ over the baseline infusion rate of $1 \mu \mathrm{g} / \mathrm{kg} / \mathrm{h}$ was recorded. Additional boluses of injection fentanyl were given when there was a $20 \%$ increase in heart rate or blood pressure associated with a painful stimulus or as deemed necessary by the attending anesthesiologist. Aneurysmal clipping was performed by the same senior neurosurgeon for all the study cases. Adverse events, time of administering the study drug, concomitant medications, and rescue analgesics were recorded for the first 24 hours postoperatively. The VAS and RSS were assessed for all patients before administration of the study drug and before and after 1 hour of administering a rescue analgesic. This was continued for the first 24 hours postoperatively after which the study drug was discontinued.

The primary per-protocol analysis included all patients who were randomized and received at least one preoperative dose of the test product and in whom a valid VAS assessment was available at four time points. The baseline covariates were compared using Fisher's exact test or Chi-square as appropriate. The decline in VAS at each time 
point was compared across the groups using a mixed effects model that incorporated a full factorial interaction term for time of assessment and treatment received. This along with the intraoperative anesthetic and analgesic requirements between trial arms formed coprimary objectives. The dose of fentanyl in $\mu \mathrm{g} / \mathrm{kg}$ (above the baseline infusion) was compared across groups using $t$-test. RSS across groups assessed postoperatively at each time point (6, 12, and 24 hours) was compared using Mann-Whitney test. Both groups were monitored for adverse events up to 24 hours after surgery and these were compared using Fisher's exact test. Blinded analysis was performed by the investigators using STATA 15.0. The data were unmasked only after the primary endpoints were assessed. This manuscript adheres to the consolidated standards of reporting trials (CONSORT) guideline.

Seventeen cases per arm were estimated to provide $80 \%$ power to detect with $5 \%$ probability of type I error, a clinically significant difference of two points in the posttreatment VAS for the pregabalin group as compared with the placebo group assuming a combined standard deviation of VAS score of 2 and an allocation ratio of 1, using a two-sided test of significance. Accounting for those who would be ineligible for the per-protocol analysis because of protocol deviations, we recruited 20 cases per arm. We did not inflate the sample size for multiple testing or intraparticipant correlation.

\section{Results}

Eighty-two patients presented at a tertiary care facility in Southern India with aSAH were screened for inclusion between October 2016 and July 2017. Forty eligible, consenting participants between 31 and 72 years of age were randomized to receive either tablet pregabalin $75 \mathrm{mg}$ BD or a placebo. Five participants who were electively ventilated postoperatively were excluded from the primary per-protocol analysis. The recruitment and follow-up is detailed in

\section{- Fig. 1.}

Patients were admitted, at median, 4 days after ictus (interquartile range [IQR]: 2-6 days) and were operated upon, on average, 21 hours after admission. The median VAS at admission was 7 (IQR: 6-8). There was no significant variation in the duration of surgery or anesthesia between the groups. Admission VAS was higher in the pregabalin group (7.61 vs. 6.71 points), and there were more men in the pregabalin arm (13 vs. 7$)$. The

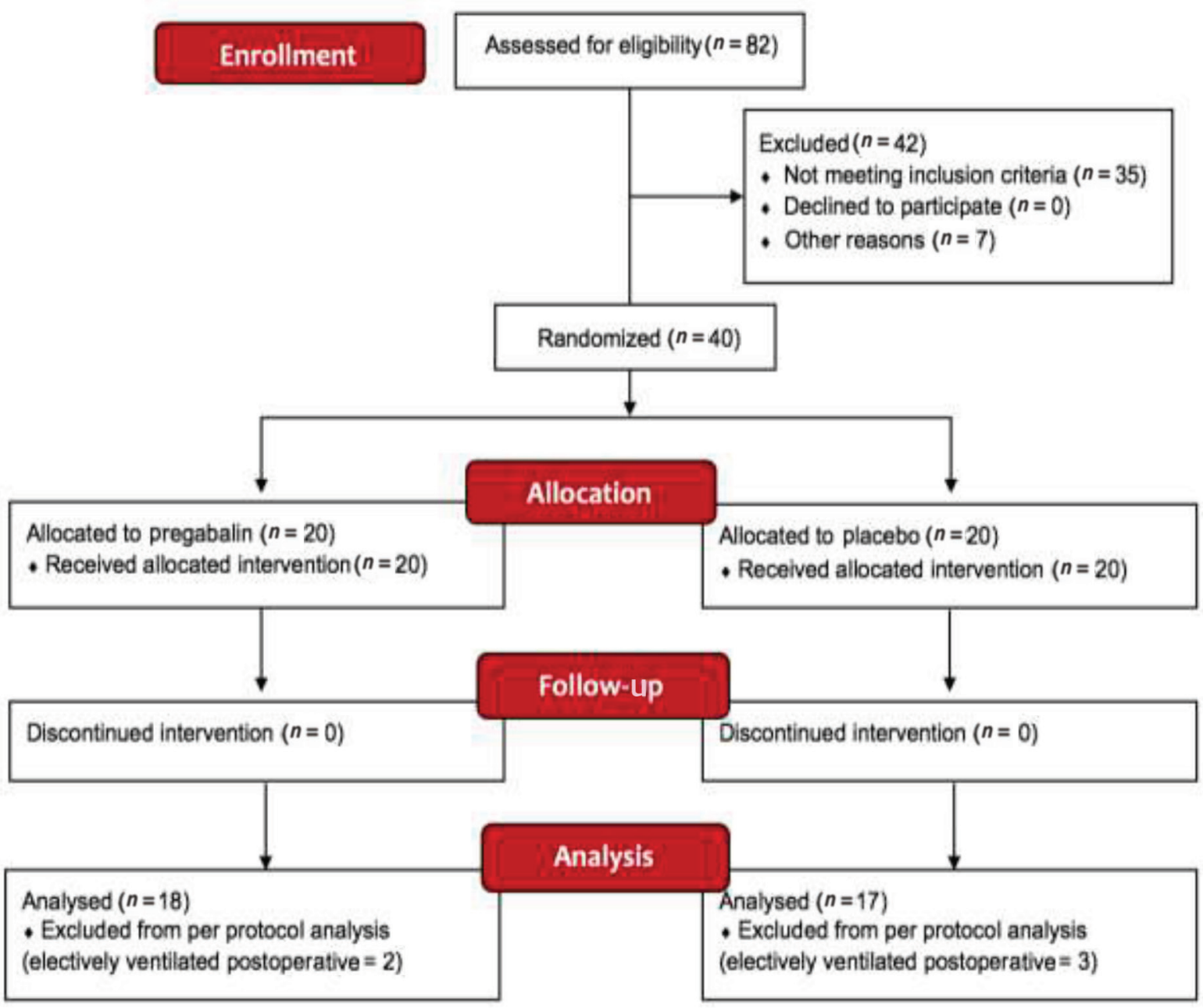

Fig.1 Consolidated standards of reporting trials (CONSORT) diagram. 
treatment groups were comparable across key baseline and treatment parameters ( $\mathbf{- T a b l e} \mathbf{1}$ ).

Headache assessed using a 10-point VAS at all postintervention time points was lower in those receiving pregabalin as compared with the placebo ( $\boldsymbol{- \text { Fig. }}$ 2A). Perioperative decline in VAS from the baseline was significantly greater among pregabalin recipients at all time points other than the 6-hour postoperative time point (- Table 2 ). The decline in VAS between admission and preinduction assessment was significantly greater in pregabalin recipients (3.61 vs. 1.76 points; $p=0.004$ ). The decline in VAS from baseline was significantly greater at the 12-hour postoperative assessment (4.33 vs. 2.76 points; $p=0.014$ ) and at the 24 -hour assessment ( 4.67 vs. 2.94 points; $p=0.007$ ) as well. At the 6-hour postoperative assessment, we did not observe a statistically significant difference in the decline in VAS (4.94 vs. 3.76; $p=0.065 ;$ - Fig. 2B).

The mean minimum alveolar concentration (MAC) requirement of sevoflurane to maintain a BIS of 40 to 60 was significantly lower in the pregabalin recipients ( 0.82 vs. $0.92 ; p=0.014 ;-$ Table 3 ). The intraoperative fentanyl requirement did not vary significantly between pregabalin and placebo recipients ( $10 \mathrm{vs} .11 .4 \mu \mathrm{g} / \mathrm{kg}, p=0.065$ ) in the primary unadjusted analysis. In a secondary, exploratory, multivariate regression analysis that adjusted for the duration of surgery and the difference in the anesthetic management between various anesthetists, we noted a statistically significant lower intraoperative fentanyl requirement of $1.64 \mu \mathrm{g} / \mathrm{kg}$ in the pregabalin recipients compared with placebo $(p=0.047)$.

Six (33\%) pregabalin recipients and nine (53\%) placebo recipients required rescue analgesics $(p=0.315)$. Pregabalin recipients required fewer doses of rescue analgesic doses than the placebo group (1.77 vs. 3.29, $p=0.005$ ).

The time to awakening was not significantly different between the groups ( 21.8 vs. 27.2 minutes, $p=0.455$ ). The RSS were similar at all time points ( - Table 4 ). The incidence of postoperative nausea and vomiting was similar across the pregabalin and placebo arms ( 0 vs. $2, p=0.356)$. One patient in each arm had vasospasm in the first 24 hours, one patient in the placebo group was reintubated and underwent a decompressive hemicraniectomy during the first 24 hours, and one patient in the placebo group developed acute onset atrial fibrillation and required cardioversion for the same. None of the adverse events led to discontinuation of the study drugs. There were no other significant adverse events that could be attributed to the drug.

\section{Discussion}

Despite presenting later than is common in Western settings, patients with acute aSAH were in considerable pain as reflected by high baseline VAS scores in both the groups (7.61 and 6.71 points). Low-dose oral pregabalin at $75 \mathrm{mg}$ twice daily decreased headache more effectively, compared with those who did not receive the drug by the time patient was being posted for aneurysm clipping. The significant decline in the VAS from the baseline to just before induction of anesthesia suggests that the drug may be effective after a median of two doses, 12 hours apart. The VAS at all postoperative time points was lower in the treatment group though this difference was not significant at the 6 -hour postoperative assessment, possibly due to the residual effects of the analgesics and anesthetics used intraoperatively in both arms. We also noted a sparing of rescue analgesic doses through the course of hospitalization, and this is an objective reiteration of the lower pain levels in those receiving pregabalin.

As postulated, there was a significant decrease in the intraoperative analgesic and anesthetic requirement in the group of patients who had received tablet pregabalin. This is reflected by the multivariate analysis which showed a higher fentanyl requirement and a higher average MAC of sevoflurane to maintain a BIS of 40 to 60 in the placebo group. The pregabalin group took 21.8 minutes to recover from anesthesia as opposed to 27.2 minute in the placebo recipients $(p=0.46)$. These findings are similar to those seen by Gupta et al who compared oral premedication with pregabalin or clonidine for hemodynamic stability during laryngoscopy and laparoscopic cholecystectomy and found that both pregabalin and clonidine decreased the need of intraoperative analgesic drug requirement. ${ }^{16}$

Table 1 Comparison of key patient characteristics across intervention arms

\begin{tabular}{|c|c|c|c|}
\hline Demographic characteristics & Pregabalin $(n=20)$ & Placebo $(n=20)$ & p-Value \\
\hline Age in years (mean $\pm S D$ ) & $53.2(9.3)$ & $53.3(7.1)$ & 0.95 \\
\hline Weight in $\mathrm{kg}($ mean \pm SD) & $67.9(10.7)$ & $60.6(17.5)$ & 0.12 \\
\hline Gender-male (\%) & $13(65)$ & $7(35)$ & 0.06 \\
\hline ASA grade $1(\%)$ & $9(45)$ & $4(20)$ & 0.09 \\
\hline Mean time to admission from ictus in $\mathrm{d}$ (SD) & $3.9(2.4)$ & $3.9(2.4)$ & 0.94 \\
\hline Mean duration of surgery in min (SD) & $356.0(60.7)$ & $347.0(74.2)$ & 0.69 \\
\hline Mean duration of anesthesia in min (SD) & $382.2(57.7)$ & $372.9(62.5)$ & 0.64 \\
\hline Mean time to awakening in min (SD) & $21.8(22)$ & $27.2(16)$ & 0.46 \\
\hline Temporary clipping: $n(\%)$ & $10(50.0)$ & $5(25.0)$ & 0.19 \\
\hline Mean duration of temporary clipping in min (SD) & $3.1(4.0)$ & $4.0(9.8)$ & 0.71 \\
\hline Mean number of preoperative study drug doses (SD) & $2.2(1.2)$ & $2.2(0.7)$ & 1.00 \\
\hline Mean number of postoperative study drug doses (SD) & $1.9(0.2)$ & $1.8(0.8)$ & 0.57 \\
\hline
\end{tabular}

Abbreviation: SD, standard deviation. 

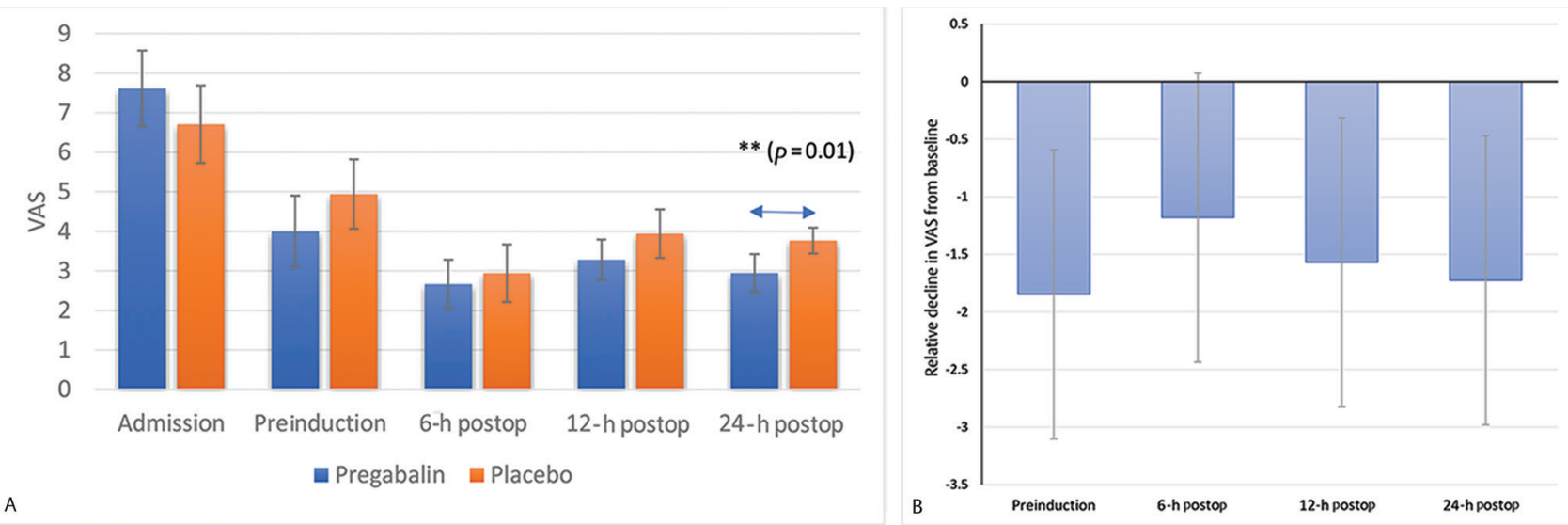

Fig. 2 (A) Headache measured on a 10-point visual analog scale at each time point across study groups. Error bars represent $95 \%$ confidence interval. (B) Excess decline in visual analog scale (from baseline) in pregabalin group at each postintervention time point. Error bars represent $95 \%$ confidence interval. Postop, postoperative; VAS, visual analog scale. ${ }^{* *}$ indicates statistically significant difference.

Table 2 Effect of pregabalin in decreasing visual analog scale from baseline at different time points

\begin{tabular}{|l|l|l|l|}
\hline Decline in VAS from baseline & Pregabalin & Placebo & Treatment effect (95\% Cl) \\
\hline Preinduction & 3.61 & 1.76 & $1.85(0.59-3.10)$ \\
\hline 6-hour postsurgery & 4.94 & 3.76 & $1.18(-0.08$ to 2.43$)$ \\
\hline 12-hour postsurgery & 4.33 & 2.76 & $1.57(0.31-2.82)$ \\
\hline 24-hour postsurgery & 4.67 & 2.94 & $1.73(0.47-2.48)$ \\
\hline
\end{tabular}

Abbreviations: $\mathrm{Cl}$, confidence interval; VAS, visual analog scale.

Note: treatment effect is the excess decline in VAS from baseline in the pregabalin recipients.

Table 3 Injection fentanyl and minimum alveolar concentration requirement of sevoflurane across intervention arms

\begin{tabular}{|l|l|l|l|}
\hline Intraoperative analgesic and anaesthetic requirement & Pregabalin & Placebo & $p$-Value \\
\hline Fentanyl requirement $(\mu \mathrm{g} / \mathrm{kg})$ & 10.0 & 11.4 & 0.065 \\
\hline Average MAC & 0.82 & 0.92 & 0.014 \\
\hline
\end{tabular}

Abbreviation: MAC, minimum alveolar concentration.

${ }^{a} \mathrm{MAC}$ of sevoflurane required to maintain bispectral index between 40 and 60 .

Table 4 Ramsay's sedation scales at different time points across intervention arms

\begin{tabular}{|l|l|l|l|}
\hline Ramsay's sedation scale & Pregabalin & Placebo & $p$-Value \\
\hline 6-hour postoperation & 2.83 & 3.01 & 0.382 \\
\hline 12-hour postoperation & 2.28 & 2.47 & 0.385 \\
\hline 24 hour postoperation & 2.06 & 2.01 & 0.339 \\
\hline
\end{tabular}

One of the key concerns with the use of pregabalin is the increased drowsiness associated with long-term use of higher doses of the drug. In this trial, we evaluated sedation using the RSS at several time points and found no evidence of increased sedation in the recipients of pregabalin. We encountered no problems with over sedation and its associated complications, such as hypoventilation, hypercarbia, hypoxia, and drowsiness, which are characterized by delayed obeying or a fall in the sensorium.

There were no related adverse events in this study nor any adverse event that required discontinuation of drugs. Nonetheless, the sample size is inadequate to make a definitive assessment of safety concerns with the drug, and larger clinical trials or meta-analysis of available data might be warranted to confirm its safety.

We acknowledge the inability to use a more objective measure of pain is a significant limitation of the study and attempted to overcome the same by standardized assessment by blinded nursing staff who were trained in making this assessment. All aSAH patients in this setting are started on antiepileptics usually tablet phenytoin $100 \mathrm{mg}$ given thrice daily and on tablet phenobarbitone $60 \mathrm{mg}$ at bedtime which could have influenced the analgesic requirement and the sedation scores across both groups. Similarly, the routine use of steroids (injection dexamethasone) with its anti-inflammatory action may have contributed to the pain relief over time. 


\section{Conclusion}

The results of this clinical trial while reiterating the utility of pregabalin $75 \mathrm{mg}$ (twice daily dosing) in reducing postoperative pain also provide evidence that, in aSAH, where patients experience excruciating pain and analgesic options are limited, pregabalin is both effective and safe as an adjunct for perioperative pain management.

\section{Funding}

None.

\section{Conflict of Interest}

None declared.

\section{References}

1 de Rooij NK, Linn FH, van der Plas JA, Algra A, Rinkel GJ. Incidence of subarachnoid haemorrhage: a systematic review with emphasis on region, age, gender and time trends. J Neurol Neurosurg Psychiatry 2007;78(12):1365-1372

2 Ingall T, Asplund K, Mähönen M, Bonita R. A multinational comparison of subarachnoid hemorrhage epidemiology in the WHO MONICA stroke study. Stroke 2000;31(5):1054-1061

3 Kassell NF, Torner JC, Haley EC, Jr. Jane JA, Adams HP, Kongable GL. The international cooperative study on the timing of aneurysm surgery. Part 1: Overall management results. J Neurosurg 1990;73(1):18-36

4 Kassell NF, Torner JC, Jane JA. Haley EC Jr., Adams HP. The international cooperative study on the timing of aneurysm surgery. Part 2: Surgical results. J Neurosurg 1990;73(1):37-47

5 Swope R, Glover K, Gokun Y, et al. Evaluation of headache severity after aneurysmal subarachnoid hemorrhage. Interdiscip Neurosurg 2014;1(4):119-122

6 Davenport R. Acute headache in the emergency department. J Neurol Neurosurg Psychiatry 2002;72(Suppl 2):ii33-ii37
7 Perry JJ, Stiell IG, Sivilotti ML, et al. High risk clinical characteristics for subarachnoid haemorrhage in patients with acute headache: prospective cohort study. BMJ 2010;341:c5204

8 Report of world federation of neurological surgeons committee on a universal subarachnoid hemorrhage grading scale. J Neurosurg 1988;68(6):985-986

9 Hunt WE, Hess RM. Surgical risk as related to time of intervention in the repair of intracranial aneurysms. J Neurosurg 1968;28(1):14-20

10 Verma V, Singh N, Singh Jaggi A. Pregabalin in neuropathic pain: evidences and possible mechanisms. Curr Neuropharmacol 2014;12(1):44-56

11 Shimony N, Amit U, Minz B, et al. Perioperative pregabalin for reducing pain, analgesic consumption, and anxiety and enhancing sleep quality in elective neurosurgical patients: a prospective, randomized, double-blind, and controlled clinical study. J Neurosurg 2016;125(6):1513-1522

12 Ulrich K, Gunreben B, Lang E, Sittl R, Griessinger N. Pregabalin in the therapy of hypnic headache. Cephalalgia 2006;26(8):1031-1032

13 Mahoori A, Noroozinia H, Hasani E, Saghaleini H. Comparing the effect of pregabalin, gabapentin, and acetaminophen on post-dural puncture headache. Saudi J Anaesth 2014;8(3):374-377

14 Taylor CP, Angelotti T, Fauman E. Pharmacology and mechanism of action of pregabalin: the calcium channel alpha2-delta (alpha2-delta) subunit as a target for antiepileptic drug discovery. Epilepsy Res 2007;73(2):137-150

15 Zeiler FA, AlSubaie F, Zeiler K, Bernard F, Skrobik Y. Analgesia in neurocritical care: An international survey and practice audit. Crit Care Med 2016;44(5):973-980

16 Gupta K, Sharma D, Gupta PK. Oral premedication with pregabalin or clonidine for hemodynamic stability during laryngoscopy and laparoscopic cholecystectomy: A comparative evaluation. Saudi J Anaesth 2011;5(2):179-184 